\title{
Philosophical Reflections on Existentialism and Phenomenology in the Films of Wong Kar-Wai
}

\author{
Huang Lianghui* \\ Wuhan University, The Yifu Teaching Building, Luojiashan Road, Wuchang District, Wuhan City, \\ Hubei Province, China \\ Email: graywong@foxmail.com \\ *Corresponding author
}

Keywords: Existentialism, Film phenomenology, Wong Kar-wai's films.

\begin{abstract}
Since the 1990s, the unique image of Wong Kar-wai's films in the pattern of world cinema is getting more and more appealing throughout the world. Through the investigation of the status, role, identity and gender of Wong Kar-wai's films, we can find that Wong Kar-wai's films are a unique landscape of the auteur film in the context of Hong Kong's post-industrial and post-colonial society. Nowadays, more and more scholars have tried to combine Wong Kar-wai's films with philosophical and aesthetic theories when the world's diverse ideologies competed and disseminated. Then, the two philosophical theories of existentialism and film phenomenology have related connections about humans and nature, which have the opportunity to have a game-like theoretical interpretation with Wong Kar-wai's films, which may better unravel the inner flow of confusion and emotional tension of his films.
\end{abstract}

\section{Wong Kar-wai's Films}

As for the search for source of love, the infatuation of sensation, and criticism of materialized reality, Wong kar-war is obviously differ from the new Hong Kong filmmakers of the 1980s and 1990s of "new wave film" and "post-new wave film", such as Hark Tsui and Ringo Lam. Unlike Chi Leung 'Jacob' Cheung and Fruit Chan, whose images are realistic and down-to-earth, Wong's films reveal a strong sense of expressionism and elitism. For Hark Tsui and Ringo Lam, they try to restore the dignity of humanity in the chivalrous world, while Wong's films tend to be aimed at the fragmentation and absurdity of life through the constant flow of reality and fiction[1]. Similarly, the films of Ann hui and Stanley Kwan are filled with the hidden femininity and feminine consciousness, and Wong's films are a kind of male perspective mixed with female gaze .

When Wong Kar-wai wrote and directed his first film As Tears Go by (in Cantonese, transliterate to Mongkok Carmen), he developed his own style according to the combination between commercial motives and picture quality. From As Tears Go by (1988) and Days of Being Wild (1990) to Chungking Express (1994); from Ashes of Time Redux (1994) and Fallen Angels (1995) to Happy Together (1997) and In the Mood for Love (1999), the ambiguous and decadent quality of nihilism and unique combination of sound and picture, as well as the huge market and box office returns of Wong's films run counter to the action spectacle and the romanticism and heroism of 
Hark Tsui and Ringo Lam; Wong's films are also different from the beautiful sentimentality and meticulous femininity in the films of Ann Hui and Stanley Kwan, and it is differ from the pure simplicity and warmth of Chi Leung 'Jacob' Cheung and Fruit Chan. "From the very beginning, Wong sought to play a distinctive role as an 'auteur' in his films - an auteur who is deeply aware of the reality of Hong Kong's post-industrial society, familiar with the game rules of the commercial film, and yet devoted to figure out the visual grammar and the language of the image, which makes him quite similar to Stanley Kubrick, Francis Ford Koppla and other 'New Hollywood Cinema' and contemporary Western film authors. In Hong Kong cinema where the tradition of modernism is largely absent, Wong's films is bound to be unique" [1]. In this way, Wong finally succeeded in developing his own film style and his own characterization.

The "Hong Kong experience" of Wong's films is closely related to the blurred image of the Hong Kong city and the mental state of the Hong Kong people in the 1990s, yet it is expressed in an unusually ambiguous and obscure way. This is not only a hopeless survival strategy but a remarkable cinematic wisdom. "Taking Hong Kong's return to China on July 1st, 1997 as the dividing line, Wong's films presented the noisy, cramped and deformed space and the extraordinary intensity of time design, the failing gain of sense of belonging in the foreign land, the fatalistic identity of status quo, the inescapable emotion and the reordering of the agenda, the beautiful and poignant perseverance of faith, and a series of other recurring narrative motives. They basically correspond to the complex journey of Hong Kong people in the face of 1997"[1]. In this way, Days of Being Wild, Ashes of Time, and In the Mood for Love can be interpreted as "Hong Kong allegory" of the 1990s. As a constructor and expresser of "Hong Kong fable", Wong Kar-wai's films belong to Hong Kong films in the strict sense before they are author films in the general sense[1].

As a representative of Hong Kong author cinema in the 1990s, the gender identity of Wong Kar-wai's films is also beyond the norm. There is an interesting phenomenon is that the male perspective is indispensable in the context of world commercial cinema, including mainstream commercial cinema in Hong Kong. In the case of Hong Kong cinema, no matter John Woo's "Hero" series or Ringo Lam's "The Storm" series are dominated by a deep-rooted male discourse; even Ann Hui and Stanley Kwan, whose female consciousness is relatively clear, show that their major works will present male perspective from time to time for the sake of a certain market orientation. So, the transcendence of Wong's films will be presented that they adopt a male point of view mixed with a female gaze[1]. This narrative strategy is typical of the gender characteristics of Wong's films, with the male protagonist's voice-over monologue interspersed with the female protagonist's voice-over monologue. Even for the general Chinese film audience and critics, the gender experimentation of Wong's films has indeed brought about an unexpected strangeness.

\section{Wong's Films: Existentialist Aesthetic Style}

The existentialist aesthetics of Wong Kar-wai's films not only present the existence of "human being", but also present the poetic realm of "being"(desain) in different artistic expressions. It highlights the situation of "human being" to the alienated society and his unique way of existence, in order to show the spiritual meaning of existentialist aesthetics. In the field of post-industrial social consciousness, the consciousness and methods of dissolution still build a path that people are eager to follow. It is Wong's conscious presentation of alienated social images that he recognizes and dissolves the phenomenon of alienation with his own emotions and actions, highlighting the essential meaning of human existence as an individual. For the technical dissolution of traditional film, Wong Kar-wai has developed a distinctive film style: the use of cinematography and zoom-up effects, as well as broken images and monologues.

The cinematic scene and techniques of Wong Kar-wai's films present a poetic beauty of art to the 
audience. For example, fancy camera movements in Days of Being Wild; the slow motion and panning shots in a dialogue "passing by tens of thousands of people every day" in Chunking Express; the martial arts scenes and the fast montage technique in Ashes of Time; the 9.5mm wide-angle lens is used to present the interpersonal relationship in the modern society in Fallen Angels; in My Blueberry Nights and The Grandmaster, the close-up shots are used to show the deep emotions of the characters, all of which provide the audience with a strong sense of visual beauty. The so-called "keep balance between dynamic and static" is the outstanding features of Wong Kar-wai's films, it is that the beauty that flows from dynamic and static is the presentation of existentialist aesthetic art. For example, in Chunking Express, the shots of Tony Chiu-Wai Leung and Faye Wong are taken by hand-held camera. The moving shots seem to be the unique style of Wong Kar-wai's films, which not only reveal the real picture, but also make people feel like they are in the scene, which is a profound interpretation of the existentialist aesthetics of "always in the process".

The long shots that flow slowly reveal a poetic mood. In The Grandmaster, the depiction of characters, still-life, and scenery is complemented by a sense of distance, creating a sense of image from Chinese ancient poem "the snow is pressing down on the pine, and the pine is straight"(大雪 压青松, 青松挺且直). In In the Mood for Love, with the slow flowing string music, Mr. Su and Ms.Zhou pass by each other in the narrow hallway, and their hidden emotions are revealed in the long slow shots, which also highlight their mutual sense of distance. [2]

The fragmentation of the picture presents the incomplete picture of the film. For example, in Fallen Angels, the distorted scenes with extremely exaggerated faces under the use of super wide-angle lens. In My Blueberry Nights, the hand-held camera shuttle back and forth towards the heroine go through restaurants, casinos and bars, and record the shaky, jumping fragmented images. In Wong's films, the shaky camera and the blurred sense of broken images seem to reflect the sense of uncertainty of "when and where", not only for the characters but also for the perception of their existence, all they can present the existential beauty to the audience.

\section{Wong's Films: Phenomenological Narratives of Cinema}

\subsection{Rejection and Acceptance}

Edmund Husserl(1859-1938), the founder of phenomenology, believes that consciousness has a temporal structure, and it is not possible to analyze the temporality of consciousness until analyzing a temporal object itself.[4] The presence of temporal objects and the temporality of consciousness are often accompanied by the location of space. Only if the temporality exists in the spatiality, can they have meaning for each other.

Then, the presentation of "distance" is the idea and the gesture that Wong's films have been holding from the beginning to the end [1]. In Wong's films, "distance" can be divided into two forms: temporal distance and spatial distance. The absurdity of space-time continuously emphasizes the distance between people, as well as the mainland and Hong Kong in Wong's films since the 1990s. The distance of absurdity also conveys the dual theme of rejection and acceptance emerging from Wong Kar-wai's films.

In fact, "distance" has become a very important element in the film of Days of Being Wild (1990). At the beginning of the film, the three minute of opening credits, can be read as a presence of the absurdity of distance between time and space [1]. On the surface, the opening credits are an explanation of the relationship between Yuddy (Leslie Cheung) and So Lai-chen (Maggie Cheung), but in fact, it is the close-up of the clock and the insertion of the picture of the tropical rain forest in the Philippines that space-time and their insurmountable distance have evolved into the main 
content[1]. Similarly, Ashes of Time, the absurdity of distance between time and space reflects the motives of the protagonist's rejection and acceptance, emotional load: Ouyang Feng (Leslie Cheung) have been missing his hometown, White Camel Mountain, and he broke up with his beloved one because of his excessive pride; But when he knew that his ex-lover died, he burned down the desert store and returned to White Camel Mountain. The spatial and temporal distance between the desert and the White Camel Mountain is not so much a physical distance between Ouyang Feng and his lover as it is a psychological distance caused by the Ouyang Feng's inability to extricate himself from his mental trauma [1].

As early as in French phenomenologist Maurice Merleau-Ponty's book The Eye and the Heart, it is described that we are not observers of the world, but people rooted in the world and living in a certain situation [3]. From the "distance" in Wong Kar-wai's films, we can feel that the individuals are in a "being" situation for the environment and the world. They are experiencing and feeling the world, and their eyes and hearts are perceiving the world. Yuddy (Ah Fei in Cantonese)is like a bird flying in the Philippine rainforest, and the intertwining of his body and nature reflects the fact that Yuddy is not going to have hopes and expectations about the world, he does not make any promise to So Lai-chen or Mimi, he just "flies" in this natural world, and even melts into nature. Wong's films have always inadvertently touched on the phenomenology of cinema, outlining the encounters between individuals. His films are filled with a strong atmosphere of emptiness lost in the city, but in reality they are all dense, ineffable individual bodily experience.

\subsection{Loss and Gain}

In Phenomenology of Perception, Maurice Merleau-Ponty(1908-1961) uses the classic example of hand touching hand to illustrate the intertwined medium of the subject and the object, and the reversibility of the subject and the object is not distinct from each other. This emotional oscillation of touching and being touched between hand and hand reflects the transformation of relationship of the seer and the seen between the self and the other. For Merleau-Ponty, the double sensation evoked by hand-touching is a representation of the archetype of the subject-object relationship: they are mutually irreplaceable, but jointly embedded in an experience of interdependence. [6]

In the genealogy of modernist spiritual culture, the emphasis on the "identity" of the self implies a search for the "ultimate" and the "root", a state of embarrassment between transient acquisition and eternal loss [1]. For Wong Kar-wai, this alienated identity of Hong Kong cinema is not only a derivative of the European and American modernist themes since the 1960s, but also the result of Hong Kong cinema's efforts to overcome schizophrenia, express self-identity and actively integrate the "Hong Kong experience" since the 1990s [1].

The dual theme of loss and gain of alienated identity almost present in all of Wong Kar-wai's films, especially Days of Being Wild[1]. The film is a spiritual allegory for the children of the fatherless/ fatherless generation in Hong Kong society in the early 1960s. In the film, Yuddy (Leslie Cheung) obtains love from So Lai-chen (Maggie Cheung), Mimi (Carina Lau), and his adoptive mother (Rebecca Pan), but he is always distraught by his dream of finding his birth mother and his urge to leave his "home". The historical absence of his biological father and mother, as well as the abnormal state of his family relations, often makes him feel like a "footless bird". As he walks out of bed bored, watching himself in front of the mirror, his voice-over monologue is filled with a tragic sense of fatalism: "I heard people say that there is a bird in the world that has no feet, it can only fly and fly, and when it gets tired of flying, it sleeps in the wind. This kind of bird only comes down to the ground once in its life, and that time is the time of death." The self-identification with the "footless bird" and the alienated identity make Yuddy experience a sense of loss from the depth of his soul [1]. 
This is a fundamental and eternal loss that nothing can make up. The loss is like the Merleau-Ponty's theory of the tentacle: they are mutually irreplaceable, but jointly embedded in an experience of interdependence [6]. As So lai-chen asks Yuddy, "Have you ever loved me or not?" Yuddy replies, "I don't know how many other women I will love in my life, and I don't know which one I love the most until the end." With the sad music and the sound of rain, the camera pans to Mimi's face, who also looks helpless and hopeless [1]. The expectation and affirmation of "finality" always pushes Yuddy towards the path of "ultimate" inquiry and "root" search, and also reinforces the decadent consciousness and tragic experience in the heart of the characters [1].

\subsection{Forgetting and Remembering}

Phenomenology believes that the object always present itself to people and become phenomenon in their consciousness, and people perceive the object presented in their consciousness and give meaningfulness to them, so that consciousness can achieve an interchangeable relationship with signifiaient and signalaient. This unification of matter and consciousness as a whole is kinds of complicated intentionality. [5]

Although Wong's films are not as filled with the value of life and the meaning of existence as modernist films such as Ingmar Bergman and Michelangelo Antonioni [1], the dual theme of forgetting and remembering through the life experience of nihilism is also the basic premise of Wong's aim to engage in an effective dialogue with Western modernist films since the 1960s.

The nihilism of life experience is not only a fundamental proposition that film makers who are in the context of modernist culture has to encounter, but also a grim reality that almost film makers who pursue the value of life and the meaning of existence have to face [1]. This existential and essential spiritual dilemma is not only manifested in the cinematic creations of classic film authors such as François Truffaut, Jean-Luc Godard, and Michelangelo Antonioni, but also in the series of films by contemporary film giants such as Andrei Tarkovsky, Krzysztof Kieslowski, and Theo Angelopoulos [1]. Strictly speaking, Wong's films are not allegories about "being". In Days of Being Wild, Fallen Angels and Ashes of Time, they are filled with themes of forgetting and remembering, and Wong express the living experience of nihilism and clearly link himself to the spiritual quest of the above-mentioned masters of cinema.

On the one hand, Days of Being Wild illustrates loss and acquisition through alienated identity. On the other hand, it confirms forgetting and remembering through the experience of nihilism[1]. In the film, So lai-chen looks through the iron fence in front of Yuddy's house after breaking up and says sadly, "I used to think that one minute would pass quickly, but actually it could be very long. One day a man pointed at his watch and told me that he would always remember me because of that one minute, and at that time I thought it sounded good! But now I look at the clock and I just tell myself that I'm going to forget about this person from this one minute on." [1] Close up shots of her face cut by the iron fence for nearly two minutes express the protagonist's state of mind of nothingness at this moment. In fact, the forgottener may have been forgotten forever, but rememberer still remembers; at the end of the film, when the one minute of April 16, 1960 at 3:00 p.m. is mentioned again, the dying Yuddy still clearly remembers "her" (So lai-chen), but he asks for his friend: "If you meet her in the future, you can tell her that I have forgotten everything, so that everyone may be better. " [1] The friend replied miserably, "I don't know if I will have the chance to meet her again, but maybe she have already forgotten me if I saw her again, ." The light flows in and out of the faces of Yuddy and his friend. The camera cuts back to Yuddy, who is already motionless and dead, leaning against the carriage seat. The dual themes of forgetting and remembering unfold tragically in the existential experience of nothingness. 


\section{Conclusions}

Under the theory of existentialism, the objective values are in danger and on the verge of collapse in the face of reality, showing people's search for new values and their desire to find the significance of their own existence. The films of Wong Kar-wai attempt to dissolve traditional values and rebuild new ones. In terms of the phenomenology of cinema, no matter the temporal structure of consciousness, the tentacle theory of hand to hand, even the intentionality of consciousness, Wong Kai-wai will generally epoche many meaningless or unrelated factors that might effect his films and concept of art. Therefore, he is so smart and hard-working that each of his films are filled with his personality, and his films have been less sharp but more refined and deliberate in recent years, exuding an aesthetic art style of existentialist and the phenomenology of cinema.

\section{References}

[1] Li Daoxin.(2001) The Spiritual Direction of Wong Kar-wai's films and Their Cultural Meaning, Contemporary Cinema.

[2] Huo Pengfei.(2015) The Aesthetic Art and Presentation of Existentialism in Wong Kar-wai's Films, Film Review. [3] Merleau-Ponty. (2007) The Eye and The Heart, Beijing: The Commercial Press.

[4] Han Lianqing.(2013) Film Phenomenology: Stigler vs. Bazin, School of Humanities, Beijing University of Aeronautics and Astronautics.

[5] Cheng Cheng.(2019) On the Problem of Encounter in Film Aesthetics, Wuhan University College of Arts.

[6] Sun Shaoyi(2012) Redefining Cinema: The Experience of Image Body and the Phenomenological Thinking of Film, Journal of Shanghai University (Social Science Edition). 